\title{
HDAC1 Silencing in Ovarian Cancer Enhances the Chemotherapy Response
}

\author{
Xinfeng Liu ${ }^{a} \quad$ Ying Yu ${ }^{b}$ Jinna Zhang ${ }^{b}$ Caixia Lu ${ }^{c} \quad$ Liming Wang ${ }^{c}$ Ping Liu ${ }^{d}$ \\ Hao Songe
}

aDepartment of Nuclear Medicine, The Affiliated Hospital of Qingdao University, Qingdao, bDepartment of Thyroid Surgery, The Affiliated Hospital of Qingdao University, Qingdao, 'Department of Gynaecology, The Affiliated Hospital of Qingdao University, Qingdao, Institute of Pathology and Southwest Cancer Center, Third Military Medical University, Chongqing, eDepartment of Radiotherapy, The Affiliated Hospital of Qingdao University, Qingdao, China

\section{Key Words}

Ovarian cancer $\bullet$ Chemoresistance $\cdot \mathrm{HDAC1} \cdot \mathrm{C}-\mathrm{Myc} \cdot \mathrm{MiR}-34 \mathrm{a}$

\begin{abstract}
Background/Aims: Cisplatin-based treatment is first-line chemotherapy for several cancers including ovarian cancer. The development of cisplatin resistance results in treatment failure, but the underlying mechanisms are not fully understood. Histone deacetylases (HDACs) are a large family of enzymes that deacetylate lysine residues on histones and nonhistone proteins. High expression of HDAC1 is associated with poor outcomes in ovarian cancer. Furthermore, resistance to chemotherapeutic agents is associated with HDAC1 overexpression in ovarian cancer cells. The goals of this study were to determine whether targeting $\mathrm{HDACl}$ can sensitize ovarian cancer cells to cisplatin and to explore the underlying mechanisms. Methods: Small interfering RNA (siRNA)-targeting HDAC1 was designed to silence HDAC1 in the cisplatin-resistant ovarian cancer cell line A2780 CDDP and its cisplatinsensitive cell line A2780. The effects of targeting HDAC1 on cell viability assay, colony formation, and apoptosis were detected. c-Myc re-expression or miR-34a inhibitors were used to examine the relationship among HDAC1, c-Myc, and miR-34a expression, which was assessed by western blot analysis and quantitative reverse transcription PCR. We established stable transfectants of A2780 CDDP/HDAC1 short hairpin RNA (shRNA) and A2780/HDAC1 shRNA. The therapeutic effectiveness of cisplatin in murine xenograft models was assessed following shRNA-mediated HDAC1 silencing in A2780 CDDP and A2780 cells. The mechanism of cell death was studied in tumor sections obtained from different mouse tumors. Results: In cisplatin-resistant A2780 CDDP cells, HDAC1 knockdown by siRNA suppressed cell proliferation, and increased apoptosis and chemosensitivity by downregulating c-Myc and upregulating miR-34a. In cisplatin-sensitive A2780 cells, HDAC1 knockdown did not affect cell proliferation and apoptosis. Cisplatin treatment activated HDAC1 and c-Myc and inactivated miR-34a. Inhibition of HDAC1 with siRNA reduced c-Myc expression, increased miR-34a expression,
\end{abstract}


Liu et al.: Targeting HDAC1 Sensitizes Ovarian Cancer Cells to Cisplatin

and sensitized A2780 cells to cisplatin-induced apoptosis. c-Myc re-expression or miR-34a targeting by miR-34a inhibitors protected cells from apoptosis or reversed cisplatin resistance following HDAC1 knockdown or/and cisplatin exposure. Finally, in vivo studies showed that targeting HDAC1 inhibited A2780 ${ }^{\mathrm{CDDP}}$-induced xenograft tumor growth but not $\mathrm{A} 2780$-induced xenograft tumor growth. Targeting HDAC1 sensitized both A2780- and A2780 CDDP-induced xenograft tumors to cisplatin treatment. Conclusions: Upregulation of HDAC1 is a crucial event in the development of drug resistance to current treatments in ovarian cancer. Thus, targeting HDAC1 by enhancing c-Myc-dependent miR-34a expression might be an effective strategy for increasing the efficacy of cisplatin treatment.

\section{Introduction}

Ovarian cancer is the fourth most common cause of cancer-related death in women because $75 \%$ of patients with ovarian cancer are diagnosed with advanced disease [1, 2]. After optimal surgical debulking of the tumor and standard chemotherapy, patients with advanced disease have a 5-year survival rate [3]. Despite the relative sensitivity of ovarian cancer to chemotherapy, clinical chemotherapeutic treatment often leads to drug resistance [4]. Development of this acquired resistance represents the major limitation to successful treatment. Consequently, there is a pressing need to identify the mechanisms underlying resistance in order to develop novel drugs to re-sensitize tumor cells to primary chemotherapy.

Histone deacetylases (HDACs) are the catalytic subunits of multiprotein complexes that deacetylate specific lysines on histone tails, resulting in the compaction of chromatin into a transcriptionally repressed state [5]. Among the different types of transcriptional repressors, HDACs are thought to be crucial enzymes in many physiological and pathological processes [6, 7]. Mammalian HDACs fall into three distinct classes based on their homology to yeast proteins; class I HDACs are homologous to the RPD3, class II HDACs are related to HDAC1, and class III HDACs are homologous to sirtuin 2. Class I HDACs (HDAC1-3 and HDAC8) share a number of structural and functional features [8]. HDACs are associated with chemoresistance in cancers such as acute myeloid leukemia [9], ovarian cancer [10-12], lung adenocarcinoma [13], oral squamous cell carcinoma [14], and colon cancer [15]. They induce chemoresistance by regulating AKT [9], Fas [10], regulator of G protein signaling 10 (RGS10) [11], miR-200b [13], B-cell lymphoma 2 [14], and Dickkopf-1 and non-canonical Wnt signaling [15].

Class I HDACs are expressed at significantly higher levels in ovarian cancer compared with normal ovarian tissues. Furthermore, the higher expression of class I HDACs (HDAC13 ) has been associated with a poor prognosis in endometrioid subtypes of ovarian and endometrial carcinomas [16]. Analysis of the effects of specific HDAC isoforms using small interfering RNA (siRNA) against HDAC1 in the ovarian carcinoma cell lines SKOV3, OVCAR3, IGROV-1, ES-2, TOV112D, A2780, and the

Cisplatin-resistant ovarian cancer cell line A2780 ${ }^{\mathrm{CDDP}}$ showed that targeting HDAC1 considerably reduced the proliferation of ovarian carcinoma cells [17]. Cacan et al [10, 11]. reported that HDAC1 overexpression in ovarian cancer cells led to the acquisition of chemoresistance, and that targeting HDAC1 increased the chemosensitivity. However, the molecular mechanisms involved in chemoresistance remain unclear.

Abnormal activation of c-Myc is thought to be involved in tumor initiation and development in many types of human cancers, including breast cancer, prostate cancer, lung carcinoma, glioblastoma, and ovarian cancer [18]. c-Myc activates and represses the transcription of discrete gene sets, leading to changes in cell proliferation, tumor progression, and maintenance [19]. High levels of c-Myc are associated with faster recurrence and poor overall survival of patients with high-grade serous ovarian cancer and cisplatin resistance in ovarian cancer cells. In addition, siRNA-based silencing of c-Myc inhibits cell growth in vitro and reduces tumor growth in xenograft models of cisplatin-resistant ovarian cancer 


\section{Cellular Physiology Cell Physiol Biochem 2018;48:1505-1518

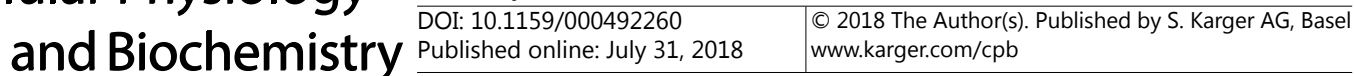

Liu et al.: Targeting HDAC1 Sensitizes Ovarian Cancer Cells to Cisplatin

$[20,21]$.

MicroRNAs (miRNAs) are a class of small noncoding RNAs involved in a wide range of processes such as proliferation, development, apoptosis, metabolism, and response to stress. Their abnormal expression has been found in many types of human tumors [22]. Among these miRNAs, miR-34a commonly functions as a tumor suppressor and is downregulated in many human cancers [23]. Furthermore, aberrant miR-34a expression has been linked to chemotherapy resistance in a variety of cancers [24-26].

c-Myc regulates multiple miRNAs, including miR-34a in many cancer cells [27-29]. In addition, downregulation of miR-34a by c-Myc is essential for tumorigenesis, as it improves tumor cell survival and promotes chemosensitity [30]. Zappasodi et al [31]. recently showed that the pan-HDAC inhibitor ITF2357 inhibited c-Myc expression in c-Myc-overexpressing human B-cell non-Hodgkin lymphomas. Adams et al [32]. reported that HDAC inhibition mediated malignant hematopoietic cell death via downregulation of c-Myc-induced apoptosis. Although targeting HDAC1 promotes apoptosis and increases chemosensitivity in cancer cells, the mechanisms of HDAC1-mediated tumorigenesis and chemoresistance are not fully understood.

In this study, we examined the biological and therapeutic effects of targeting HDAC1 by siRNA or short hairpin RNA (shRNA) in the cisplatin-resistant ovarian cancer cell line $A 2780^{\mathrm{CDDP}}$ and its cisplatin-sensitive A2780 cell line in vitro and in vivo. We identified the HDAC1/c-Myc/miR-34a pathway as a novel driver of cisplatin resistance in ovarian cancer cells. Thus, targeting HDAC1 through enhancing c-Myc-dependent miR-34a expression might be an effective strategy for increasing the efficacy of cisplatin treatment.

\section{Materials and Methods}

\section{Cell culture}

Human cisplatin-resistant ovarian cancer A2780 ${ }^{\mathrm{CDDP}}$ cells and cisplatin-sensitive A2780 cells were purchased from American Type Culture Collection (Shanghai, China). Cells were cultured in RPMI-1640 medium supplemented with $10 \%$ heat-inactivated fetal bovine serum, $100 \mathrm{U} / \mathrm{mL}$ penicillin, $100 \mu \mathrm{g} / \mathrm{mL}$ streptomycin, and $2.5 \mu \mathrm{g} / \mathrm{mL}$ amphotericin B. Cells were maintained at $37^{\circ} \mathrm{C}$ in a humidified incubator with $5 \% \mathrm{CO}_{2} / 95 \%$ air.

\section{shRNA-mediated knockdown of HDAC1}

shRNA constructs targeting HDAC1 and scrambled control were purchased from Open Biosystems (Lafayette, CO, USA). Transfection of A2780 ${ }^{\mathrm{CDDP}}$ and A2780 cells with lentiviral particles containing HDAC1 shRNA and verification of the percent knockdown were performed according to the manufacturer's instructions. Briefly, bacterial glycerol stocks containing the lentiviral plasmid vector pLKO.1 with shRNA inserts against clusterin, an empty vector, or a scrambled shRNA control were obtained from Sigma-Aldrich (Poole, UK). Lentivirus-transfected 293T cells were incubated at $37^{\circ} \mathrm{C}$ for $48 \mathrm{~h}$ before the resulting lentiviral particles were harvested by centrifugation and concentrated using the Clontech Lenti-X Concentrator Kit (Lonza, Wokingham, UK). Then concentrated virus was added to A2780 ${ }^{\mathrm{CDDP}}$ and A2780 cells and incubated for $48 \mathrm{~h}$. Lentivirus-transduced cells were selected by addition of puromycin $(1 \mu \mathrm{g} / \mathrm{mL})$ to the cell culture for 2 weeks to produce stable HDAC1 shRNA-transfected vector. The relative expression of HDAC1 was subsequently assessed by quantitative reverse transcription PCR (qRT-PCR) and western blotting.

\section{Transient siRNA-mediated knockdown of HDAC1}

$\mathrm{A} 2780^{\mathrm{CDDP}}$ and A2780 cells were seeded at a density of $1 \times 10^{5}$ cells/well in 24 -well plates. After a 3-h incubation at $37^{\circ} \mathrm{C}$, the cells were transfected with $50 \mathrm{nM}$ HDAC1 siRNA or scrambled siRNA (Ambion, Austin, TX, USA) using Lipofectamine 2000 (Invitrogen, Carlsbad, CA, USA) according to the manufacturer's instructions. After a 48 -h incubation at $37^{\circ} \mathrm{C}$, the relative expression of HDAC1 was assessed by qRT-PCR and western blotting. 


\section{Cellular Physiology Cell Physiol Biochem 2018;48:1505-1518 and Biochemistry Published online: July 31, $2018 \quad \begin{aligned} & \text { DOI: } 2018 \text { The Author(s). Published by S. Karger AG, Basel } \\ & \text { www.karger.com/cpb }\end{aligned}$}

Liu et al.: Targeting HDAC1 Sensitizes Ovarian Cancer Cells to Cisplatin

Plasmid constructs and stable transfection

c-Myc cDNA amplified from A2780 ${ }^{\mathrm{CDDP}}$ cells was inserted into the pcDNA3.1 vector (Thermo Fisher Scientific, Waltham, MA, USA). These lentiviral vectors were transfected into 293T cells with other packaging plasmids to generate viruses that were used to obtain A2780 ${ }^{\mathrm{CDDP}}$ and A2780 cells expressing c-Myc. The stably transfected cells were generated by hygromycin B selection. The plasmids were generated using standard techniques and verified by sequencing. To attain cells stably expressing miR-34a inhibitory sequences, A2780 ${ }^{\mathrm{CDDP}}$ or A2780 cells were transduced with the anti-miR-34a lentiviruses (System Biosciences, Mountain View, CA, USA) produced in 293Ta cells according to the manufacturer's instructions. Two days after transduction, selection with $0.5 \mu \mathrm{g} / \mathrm{mL}$ puromycin was performed and $95 \%$ transduction efficiency, evaluated by flow cytometry analysis, was generally obtained in A2780 ${ }^{\mathrm{CDDP}}$ and A2780 cells. After 4-5 days of puromycin selection, $A 2780^{\mathrm{CDDP}}$ and $\mathrm{A} 2780$ cells were ready for subsequent experiments.

\section{Treatment with cisplatin}

A2780 ${ }^{\mathrm{CDDP}}, \mathrm{A} 2780$ cells, or stable pcDNA3.1 c-Myc cDNA-transfected A2780 $\mathrm{CDDP}$ and A2780 cells or stable anti-miR-34a lentivirus-transfected $A 2780^{\mathrm{CDDP}}$ and $\mathrm{A} 2780$ cells were plated in 96-well cell culture plates at a concentration of $1 \times 10^{4}$ cells/well. Cells were transfected with $50 \mathrm{nM}$ HDAC1 siRNA or scrambled siRNA. After $48 \mathrm{~h}, 0-3 \mu \mathrm{g} / \mathrm{mL}$ cisplatin was added to each of the cells. Treatment wells were set up in triplicate, and the cells were incubated with cisplatin for an additional $48 \mathrm{~h}$. After treatment, the cells were washed with phosphate-buffered saline (PBS), and $100 \mu \mathrm{L}$ medium was added back to the wells. Cell viability, apoptosis, and protein and messenger RNA (mRNA) expression were detected.

\section{Colony-forming assay}

$\mathrm{A} 2780^{\mathrm{CDDP}}$ and A2780 cells were allowed to adhere for 4-6 h, and were then transfected with HDAC1 siRNA or mock-treated for $24 \mathrm{~h}$, exposed to $3 \mu \mathrm{g} / \mathrm{mL}$ cisplatin for $1 \mathrm{~h}$, washed with PBS, and then re-seeded in $60 \mathrm{~mm}$ dishes at a density of 150 cells per dish. After 7-9 days, the cells were fixed in ethanol and stained with crystal violet, after which colonies $>50$ cells were counted. Groups of $>50$ cells were scored as colonies. To detect the effects of c-Myc or miR-34a on colony formation, cells stably expressing c-Myc or anti-miR34a cells were plated at a density of 1000 cells per dish in $60 \mathrm{~mm}$ dishes, allowed to adhere overnight, transfected with HDAC1 siRNA or mock-treated for $24 \mathrm{~h}$, and exposed to $3 \mu \mathrm{g} / \mathrm{mL}$ cisplatin for $1 \mathrm{~h}$. After 14 days, the cells were fixed in ethanol and stained with crystal violet, after which colonies $>50$ cells were counted. Groups of $>50$ cells were scored as colonies.

\section{Cell proliferation assay}

A2780 ${ }^{\mathrm{CDDP}}, \mathrm{A} 2780$, and cells stably expressing c-Myc cDNA or anti-miR-34a were seeded in 96-well plates at an initial density of $2 \times 10^{3}$, incubated for $24 \mathrm{~h}$, transfected with HDAC1 siRNA or mock-treated for $24 \mathrm{~h}$, and exposed to $3 \mu \mathrm{g} / \mathrm{mL}$ cisplatin for $1 \mathrm{~h}$. After drug treatment, cell cultures were incubated for another $24 \mathrm{~h}$. At the end of the growth period, the cells were washed with PBS, fixed in $3.7 \%$ formaldehyde for $30 \mathrm{~min}$, and stained with $1.0 \%$ methylene blue for $30 \mathrm{~min}$. Plates were rinsed in running water and then left to dry. A total of $100 \mu \mathrm{L}$ solvent (10\% acetic acid, 50\% methanol, and 40\% water) was added to each well to dissolve the cells, and the optical density of the released color was read at $660 \mathrm{~nm}$. The relative cell viability was calculated with the values of mock-treated cells set as $100 \%$.

\section{Assessment of in vitro cell apoptosis}

Apoptosis was measured with the FITC Apoptosis Detection Kit (BD Biosciences, San Jose, CA, USA), which uses Annexin V and propidium iodide (PI) as apoptotic and necrotic markers, respectively. Briefly, cells in different groups $\left(1 \times 10^{5}\right)$ were collected, washed, and re-suspended in $1 \mathrm{X}$ binding buffer. Cells were incubated for 10 min with Annexin V and/or PI according to the manufacturer's instructions. After the addition of $500 \mu \mathrm{L} 1 \mathrm{X}$ binding buffer, apoptotic cells were analyzed using the FACSCalibur flow cytometer (BD Biosciences). CellQuest ${ }^{\mathrm{TM}}$ Pro software (BD Biosciences) was used to determine the number of apoptotic and necrotic cells. 


\section{Cellular Physiology Cell Physiol Biochem 2018;48:1505-1518 and Biochemistry Published online: July 31, $2018 \quad \begin{aligned} & \text { DOI: 10.1159/000492260 } \\ & \begin{array}{l}\text { (c) } 2018 \text { The Author(s). Published by S. Karger AG, Basel } \\ \text { www.karger.com/cpb }\end{array}\end{aligned}$}

Liu et al.: Targeting HDAC1 Sensitizes Ovarian Cancer Cells to Cisplatin

\section{Western blot analysis}

Cells in different groups were washed with PBS and then lysed in lysis buffer containing $50 \mathrm{mM}$ Tris$\mathrm{HCl}$ (pH 7.5), $150 \mathrm{mM} \mathrm{NaCl}, 1 \mathrm{mM}$ EDTA, $1 \mathrm{mM} \mathrm{MgCl}_{2}$, and $0.5 \%$ Triton X-100. Lysates were cleared by centrifugation at $13,000 \times \mathrm{g}$ for $20 \mathrm{~min}$ at $4^{\circ} \mathrm{C}$ and analyzed by western blotting. Protein samples were separated by sodium dodecyl sulfate polyacrylamide gel electrophoresis, transferred to a polyvinylidene difluoride membrane, and probed with the following antibodies: HDAC1, c-Myc (Cell Signaling Technology, Shanghai, China), and glyceraldehyde-3-phosphate dehydrogenase (GAPDH; Santa Cruz, Dallas, TX, USA). Proteins were detected by chemiluminescence.

\section{Quantitative reverse transcription polymerase chain reaction}

Total RNA (100 ng) was reverse-transcribed to cDNA using the High Capacity cDNA Archive Kit (Life Technologies, Carlsbad, CA, USA). TaqMan PCR was performed using the TaqMan Fast Advanced PCR Master Mix and TaqMan Gene Expression Assays (all reagents from Life Technologies) using the 7900HT Fast Real-Time PCR System (Applied Biosystems, Foster City, CA, USA). Assays were performed in triplicate. Gene expression profiling was achieved using the comparative cycle threshold (CT) method of relative quantitation using GAPDH as a housekeeping gene. To normalize data, $\Delta \Delta \mathrm{CT}$ was calculated for each sample using the mean of its $\Delta \mathrm{CT}$ values subtracted from the mean $\Delta \mathrm{CT}$ value measured in the control sample, set as a calibrator; relative quantitation (RQ) value was expressed as $2^{-\Delta \Delta C T}$. MiR-34a detection by quantitative reverse transcription PCR (qRT-PCR) was performed using TaqMan MicroRNA Assays (Life Technologies). Individual reverse transcription reactions (5 ng total RNA each target) were performed using the Taqman MicroRNA Reverse Transcription Kit and miRNA-specific looped-primers. TaqMan PCR was performed in triplicate using the 7900HT Fast Real-Time PCR System (Applied Biosystems). miRNA expression RQ data were calculated as reported above using U6 snRNA as the housekeeping control.

\section{Tumor xenograft model and treatment}

The stable HDAC1 shRNA-transfected A2780 ${ }^{\mathrm{CDDP}}$ or stable HDAC1 shRNA-transfected A2780 cell suspension $\left(2 \times 10^{6}\right)$ was mixed with matrigel (BD Biosciences) at a 1:1 volume ratio, and the mixture was subcutaneously injected into the flanks of 4-6-week-old nude female mice. Approval of the study protocol was obtained and supervised by the Affiliated Hospital of Qingdao University Subcommittee on Research Animal Care (Shandong, China). When the tumor volume in mice reached approximately $100 \mathrm{~mm}^{3}$, the mice (six per group) were randomly assigned to control or treatment groups, and treated with an intraperitoneal injection of saline or cisplatin $(160 \mu \mathrm{g} /$ injection $)$ once a week for 4 weeks. The health of the mice was monitored daily during the treatment period. Tumor size was measured by calipers every other day. The tumor volume was calculated with the formula: length $\times$ width $^{2} \times 0.52$. After 5 weeks, all surviving mice were euthanized with $\mathrm{CO}_{2}$ overdose and evaluated macroscopically for the presence of orthotopic tumors. Part of the tissue was stored frozen at $-80^{\circ} \mathrm{C}$ until processing. The other tissue specimens were fixed in formalin for paraffin embedding for subsequent histological analysis and immunohistologic staining.

\section{Immunohistologic staining}

Tissues were fixed in formalin, embedded in paraffin, sectioned ( $5 \mu \mathrm{m})$ and stained with hematoxylin and eosin. Immunostaining was performed according to standard techniques using a polyclonal antibody specific for human HDAC1 and c-Myc. Detection was performed using a horseradish peroxidase-conjugated secondary antibody followed by chromogenic detection using DAB (Sigma, St. Louis, MO, USA) as the substrate. Cells positive for HDAC1 and c-Myc were counted in five high-power fields.

Terminal deoxynucleotidyl transferase dUTP nick end labeling staining

Terminal deoxynucleotidyl transferase dUTP nick end labeling (TUNEL) staining was performed using the In Situ Cell Death Detection Kit (Roche Diagnostics, Risch-Rotkreuz, Switzerland). Slides of paraffinembedded tissues of resected tumors in mice were dewaxed and rehydrated according to standard protocols. Then, tissue sections were incubated for $20 \mathrm{~min}$ at room temperature with proteinase $\mathrm{K}(20 \mu \mathrm{g} / \mathrm{mL})$. After rinsing the slides twice with PBS, cells were incubated in $50 \mathrm{~mL}$ TUNEL reaction mixture for $1 \mathrm{~h}$ at $37^{\circ} \mathrm{C}$ in the dark. Next, $50 \mathrm{~mL}$ DAPI was added and incubated for $2 \mathrm{~min}$ at room temperature. Cells were imaged by fluorescent microscopy at $488 \mathrm{~nm}$ excitation and $530 \mathrm{~nm}$ emission. Cells exhibiting green fluorescence were defined as TUNEL-positive apoptotic cells. 


\section{Statistical analysis}

Statistical analysis was conducted using SPSS 16.0 for Windows (SPSS, Chicago, IL, USA). The twotailed Student's $t$-test was used for the analysis of continuous variables. $P<0.05$ was considered statistically significant. We determined differences among more than three groups using non-repeated measures analysis of variance. Results are expressed as the mean \pm standard deviation and $P<0.05$ was considered statistically significant.

\section{Results}

\section{HDAC1 expression in ovarian cancer cells}

We first performed growth inhibition experiments to confirm the responsiveness of the ovarian parental cancer cell line A2780 and its cisplatin-resistant counterpart A2780 ${ }^{\mathrm{CDDP}}$. As expected, the resistant cells had no response while the sensitive cells had a dose-dependent response (Fig. 1A). Next, we used qRT-PCR and western blotting to compare the HDAC1 mRNA and protein expression levels, respectively, in A2780 and A2780 ${ }^{\mathrm{CDDP}}$ cells (Fig. 1B, C). HDAC1 was more highly expressed in A2780 ${ }^{\mathrm{CDDP}}$ cells compared with A2780 cells. These results suggest that HDAC1 is a clinically relevant target for patients with cisplatin-resistant ovarian cancer.

Fig. 1. Effects of HDAC1 on ovarian cancer cell growth, viability, and apoptosis. A, Human cisplatinresistant ovarian cancer $\mathrm{A} 2780^{\mathrm{CDDP}}$ cells and its cisplatin-sensitive A2780 cells were treated with cisplatin at concentrations of $0,0.1$, 1.0 , and $3 \mu \mathrm{g} / \mathrm{mL}$. Cell viability was calculated $48 \mathrm{~h}$ after cisplatin treatment as previously described. The mean \pm standard error of the mean (SEM) are shown; B, $\mathrm{A} 2780^{\mathrm{CDDP}}$ and $\mathrm{A} 2780$ cells were transfected with HDAC1 siRNA (0-100 nM) for $48 \mathrm{~h}$. HDAC1 mRNA expression was detected by the qRT-PCR assay; C, HDAC1 protein was detected by western blot analysis as described in the "Materials and Methods" section. GAPDH was used as a loading control. D, Cell viability was detected by the MTT assay; E, The percent of clonogenicity was calculated relative to the control siRNA in A2780 ${ }^{\mathrm{CDDP}}$ cells. F, The percent of clonogenicity was calculated relative to control siRNA in A2780 cells. G, H, The distribution of apoptotic A2780 and $\mathrm{A} 2780^{\mathrm{CDDP}}$ cells measured by Annexin V and PI staining using flow cytometry. Representative dot plots from one $t$ of three independent experiments are shown. The mean \pm SEM are shown for three independent experiments $\left({ }^{*} \mathrm{P}<0.05,{ }^{* *} \mathrm{P}<0.01\right)$.

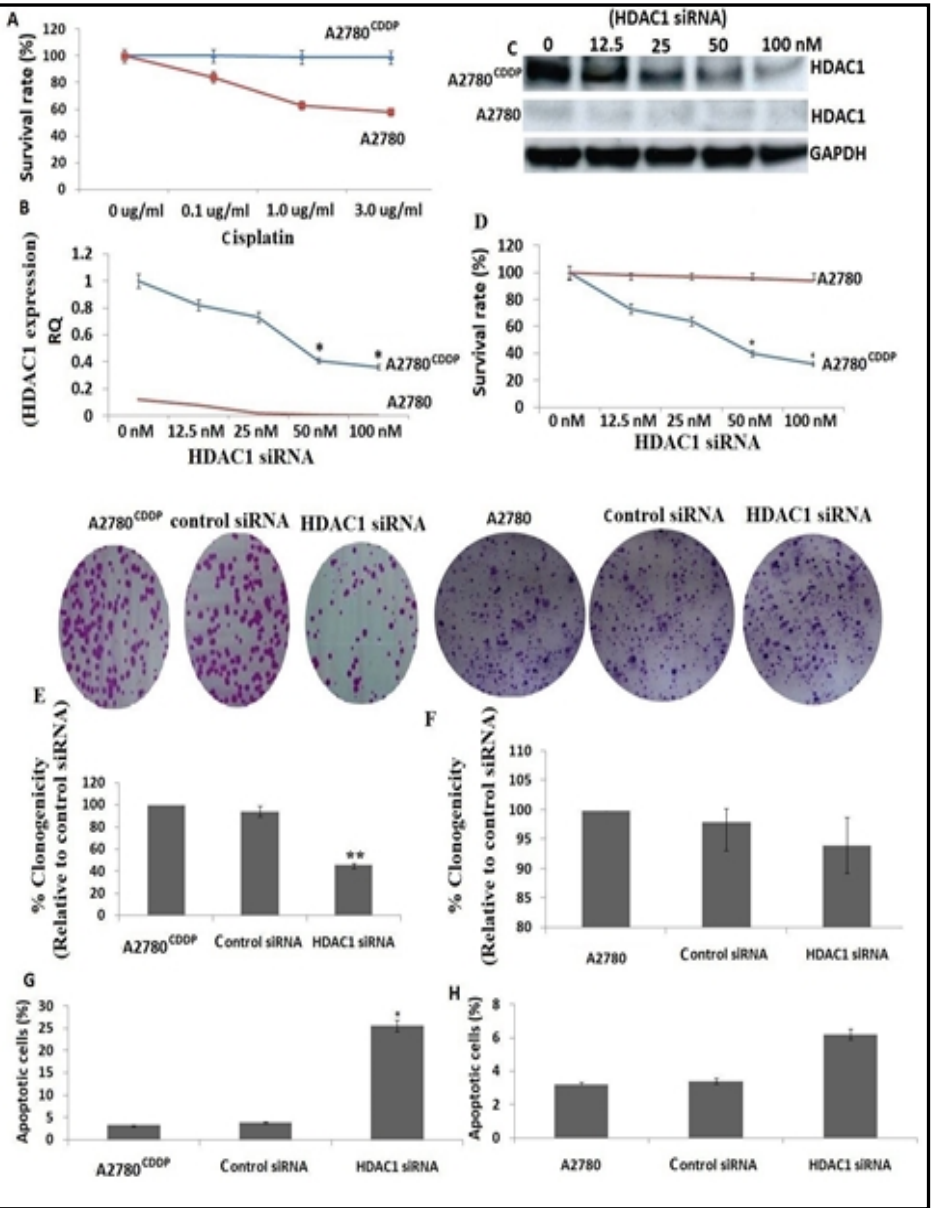


In vitro knockdown of HDAC1 affects cisplatin-resistant but not cisplatinsensitive cells

Next, we examined the biological effects of HDAC1 silencing in cisplatin-resistant A2780 ${ }^{\text {CDDP }}$ cells. The qRT-PCR and western blotting experiments demonstrated that siRNA targeting HDAC1 reduced HDAC1 mRNA and protein levels in the A2780 ${ }^{\mathrm{CDDP}}$ cisplatin-resistant ovarian cancer cell line (Fig. 1B, C). Dose-dependent inhibition of cell viability was observed after $48 \mathrm{~h}$ of HDAC1-targeted siRNA treatment (Fig. 1D). The inhibitory effects on cell viability were observed at doses as low as 12.5 nM HDAC1siRNA (Fig. 1D). Treatment with HDAC1siRNA also induced long-term effects in cell growth as evident in colony formation assays. Transfection of HDAC1-siRNA in A2780 CDDP cells significantly reduced (55\% reduction, $\left.{ }^{* *} P<0.01\right)$ the number of colonies formed after 7 days in culture compared with control siRNA-transfected cells (Fig. 1E). Conversely, HDAC1 silencing in A2780 cisplatin-sensitive ovarian cancer cells, which express low levels of HDAC1, induced negligible changes in cell viability (Fig. 1D) and colony formation (Fig. $1 \mathrm{~F})$. Levels of apoptosis were determined by the Annexin-V FITC/PI assay. The results showed that transfection of A2780 CDDP cells with HDAC1 siRNA resulted in a significantly higher number of apoptotic cells $(23.6 \pm 3.8 \%)$ compared with control siRNA $(3.2 \pm 0.9 \%, P<$ 0.05; Fig. 1G). HDAC1 downregulation by siRNA did not exhibit any detectable effects compared with control siRNA in A2780 cells $(P<0.05$; Fig. $1 \mathrm{H})$.

Cisplatin treatment induces HDAC1 expression in A2780 cells

A2780 and A2780 ${ }^{\mathrm{CDDP}}$ cells were treated with cisplatin $(0,0.01,0.1,1.0$, and $3 \mu \mathrm{g} / \mathrm{mL})$ for $48 \mathrm{~h}$. The mRNA and protein expression levels of HDAC1 were detected by qRT-PCR and western blotting. The results showed that A2780 cells had a dose-dependent increase in HDAC1 mRNA and protein expression (Fig. 2A, B), while in A2780 ${ }^{\mathrm{CDDP}}$ cells, which expresses high endogenous HDAC1 levels, cisplatin treatment induced negligible changes in HDAC1 mRNA and protein expression (Fig. 2A, B).

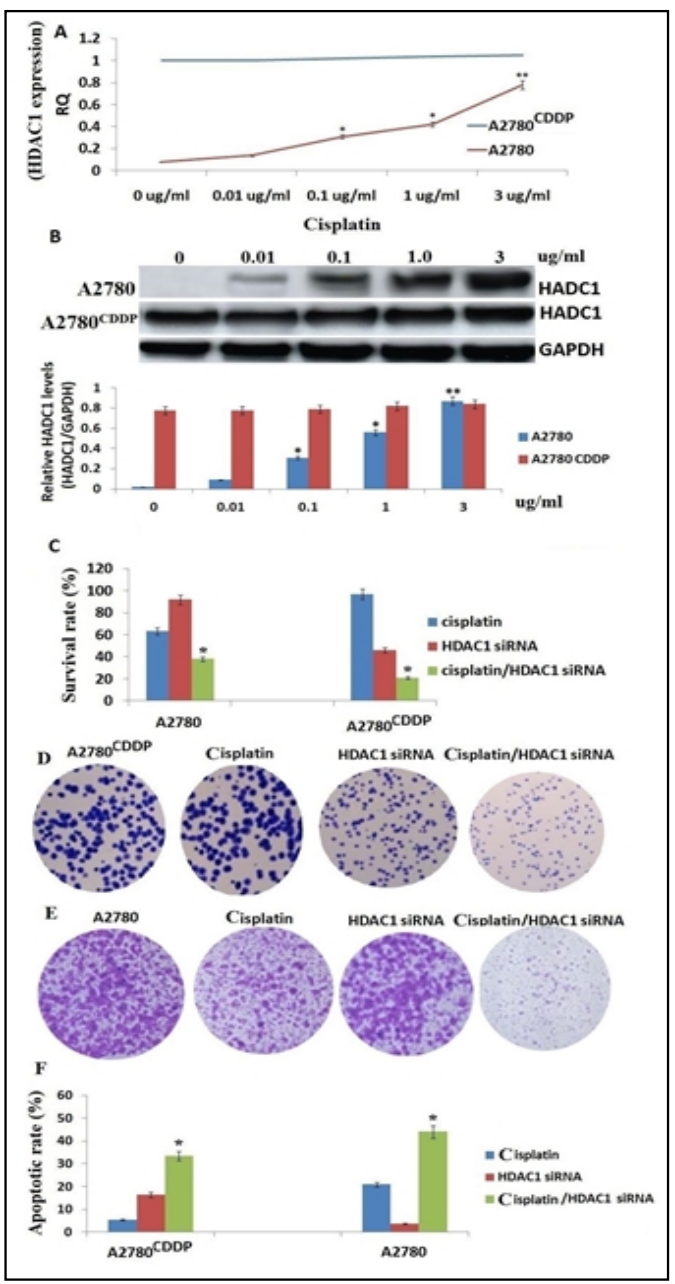

Fig. 2. HDAC1 silencing enhances the sensitivity of A2780 and A2780 ${ }^{\text {CDDP }}$ cells to cisplatin. A, A2780 and $\mathrm{A} 2780^{\mathrm{CDDP}}$ cells were treated with cisplatin at concentrations of $0.01,0.1,1.0$, and $3 \mu \mathrm{g} / \mathrm{mL}$ for $48 \mathrm{~h}$. HDAC1 mRNA was detected by the qRT-PCR assay; B, A2780 and A2780 ${ }^{\mathrm{CDDP}}$ cells were treated with cisplatin at concentrations of $0.01,0.1,1.0$, and $3 \mu \mathrm{g} / \mathrm{mL}$ for $48 \mathrm{~h}$. HDAC1 protein was detected by western blotting. C, A2780 and A2780 ${ }^{\mathrm{CDDP}}$ cells were treated with cisplatin $(3 \mu \mathrm{g} / \mathrm{mL})$ or/and $50 \mathrm{nM}$ HDAC1 siRNA for $48 \mathrm{~h}$, and cell viability was detected by the methylene blue assay; D, A2780 ${ }^{\text {CDDP }}$ cells were treated with cisplatin $(3 \mu \mathrm{g} /$ $\mathrm{mL}$ ) or/and $50 \mathrm{nM}$ HDAC1 siRNA for 7 days, and the number of colonies formed was detected; $\mathrm{E}$, A2780 cells were treated with cisplatin $(3 \mu \mathrm{g} / \mathrm{mL}$ ) or/and $50 \mathrm{nM}$ HDAC1 siRNA for 7 days, and the number of colonies formed was detected; F, A2780 and $\mathrm{A} 2780^{\mathrm{CDDP}}$ cells were treated with cisplatin (3 $\mu \mathrm{g} / \mathrm{mL}$ ) or/and $50 \mathrm{nM}$ HDAC1 siRNA for $48 \mathrm{~h}$, and cell apoptosis was detected using the FACSCalibur flow cytometer $\left({ }^{*} \mathrm{P}<0.01\right)$. 
HDAC1 silencing enhances the sensitivity of $A 2780$ and $A 2780^{C D D P}$ cells to cisplatin

A2780 and A2780 ${ }^{\mathrm{CDDP}}$ cells were was transfected with HDAC1 siRNA in the presence or absence of cisplatin for $48 \mathrm{~h}$ prior to the cell proliferation assay. As shown in Fig. 2C, while the viability of $\mathrm{A} 2780$ and $\mathrm{A} 2780^{\mathrm{CDDP}}$ cells was reduced to $37 \%$ and $2 \%$, respectively, due to the potent cytoxicity of $3 \mu \mathrm{g} / \mathrm{mL}$ cisplatin, the effects of $50 \mathrm{nM}$ HDAC1 siRNA combined with drug exposure resulted in a $68 \%$ and $79 \%$ reduction in cell viability, respectively. The colony formation assay showed that cisplatin $(3 \mu \mathrm{g} / \mathrm{mL})$ combined with $50 \mathrm{nM}$ HDAC1 siRNA significantly reduced the number of colonies formed after 7 days in culture compared with HDAC1 siRNA or cisplatin alone in both A2780 and A2780 ${ }^{\mathrm{CDDP}}$ cells (Fig. 2D, E). Treatment with cisplatin $(0,0.01,0.1$, and $1.0 \mu \mathrm{g} / \mathrm{mL})$ combined with $50 \mathrm{nM}$ HDAC1 siRNA led to the same results as above (date not shown). In addition, HDAC1 silencing increased the apoptotic response to treatment of A2780 and A2780 ${ }^{\mathrm{CDDP}}$ cells with $3 \mu \mathrm{g} / \mathrm{mL}$ cisplatin (Fig. $2 \mathrm{~F}$ ). Thus, the combination of siRNA and cisplatin induced more cell death than drug or siRNA treatment alone.

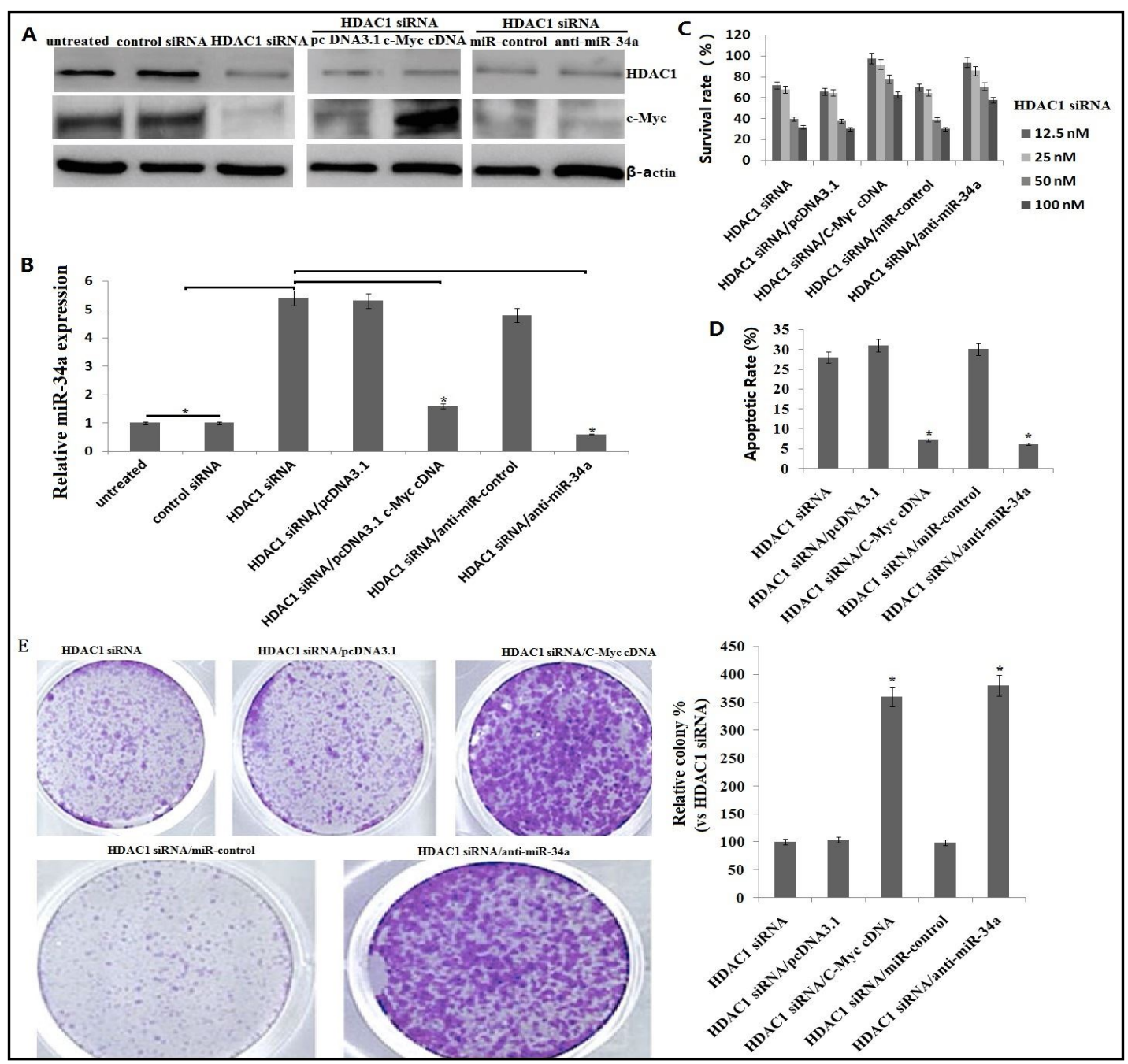

Fig. 3. HDAC1 silencing inhibits $A 2780^{\mathrm{CDDP}}$ cell growth and colony formation via the c-Myc/miR-34a pathway. A2780 ${ }^{\mathrm{CDDP}}$ cells were transfected with HDAC1 siRNA (50 nM) for $48 \mathrm{~h}$, or cells stably expressing c-Myc or anti-miR-34a or t control-transfected A2780 ${ }^{\mathrm{CDDP}}$ cells were transfected with HDAC1 siRNA (50 nM) for $48 \mathrm{~h}$. HDAC1 and c-Myc expression was detected by western blot analysis; A, miR-34a was detected by qRT-PCR; B, Cell viability was detected by the methylene blue assay; C, Cell apoptosis was detected using the FACSCalibur flow cytometer; D, The number of colonies formed was detected; E, vs. HDAC1 $\operatorname{siRNA}\left({ }^{*} \mathrm{P}<0.01\right)$. 
In vitro knockdown of HDAC1 reduced c-Myc expression and increased c-Myc-dependent miR-34a expression in $A 2780^{C D D P}$ cells

Targeting HDAC1 by transfection of A2780 ${ }^{\mathrm{CDDP}}$ cells with HDAC1 siRNA ( $50 \mathrm{nM}$ ) markedly decreased c-Myc protein expression as detected by western blotting (Fig. 3A), and increased miR-34a expression by the qRT-PCR assay (Fig. 3B). In addition, transfection of a plasmid containing c-Myc increased c-Myc expression (Fig. 3A) and efficiently inhibited miR-34a expression in HDAC1 siRNA-transfected A2780 ${ }^{\mathrm{CDDP}}$ cells (Fig. 3B). However, targeting miR34a expression did not affect c-Myc expression (Fig. 3A) or HDAC1 expression (Fig. 3B) in HDAC1 siRNA-transfected A2780 ${ }^{\mathrm{CDDP}}$ cells.

Targeting HDAC1 affects A2780 ${ }^{C D D P}$ cell growth and colony formation via the c-Myc/miR34a pathway

The abovementioned data showed that targeting HDAC1 affects A2780 ${ }^{\mathrm{CDDP}}$ cell growth, apoptosis, and colony formation. Furthermore, targeting HDAC1 reduced c-Myc expression and increased c-Myc-dependent miR-34a expression in A2780 ${ }^{\mathrm{CDDP}}$ cells. Next, we examined whether the c-Myc/miR-34a pathway is associated with A2780 ${ }^{\mathrm{CDDP}}$ cell growth and apoptosis in response to HDAC1 downregulation. Re-expression of c-Myc by c-Myc cDNA transfection (Fig. 3A) or miR-34a silencing by anti-miR-34a transfection (Fig. 3A) rescued A2780 ${ }^{\mathrm{CDDP}}$ cell growth (Fig. 3C), apoptosis (Fig. 3D), and colony formation (Fig. 3E) after HDAC1 siRNA transfection.

Targeting HDAC1 sensitizes A2780 and A2780 ${ }^{C D D P}$ cells to cisplatin via the c-Myc/miR-34a pathway

The abovementioned cytotoxic effects were greatly potentiated by the combination of HDAC1 silencing and cisplatin ( $3 \mu \mathrm{g} / \mathrm{mL})$ in both A2780 and A2780 CDDP cells. However, re-expression of c-Myc or miR-34a silencing was sufficient to rescue the viability (Fig. 4A), apoptosis (Fig. 4B), and colony formation (Fig. 4C), indicating that silencing HDAC1 sensitizes $\mathrm{A} 2780$ and $\mathrm{A} 2780^{\mathrm{CDDP}}$ cells to cisplatin via the c-Myc/miR-34a pathway.

Inhibition of HDAC1 sensitizes ovarian cancer cells to cisplatin in vivo

To determine whether systemic therapy with HDAC1 silencing and cisplatin could inhibit tumor growth in animals, we established stable HDAC1 shRNA-transfected A2780 and $A 2780^{\mathrm{CDDP}}$ ovarian cancer xenografts in SCID mice. We found that mice in all treatment groups developed tumors. As shown in Fig 5A and 5B, HDAC1 shRNA transfection alone significantly inhibited tumor growth in A2780 ${ }^{\mathrm{CDDP}}$ ovarian cancer xenografts but not in A2780 ovarian cancer xenografts compared with the untreated control. In addition, cisplatin treatment alone significantly inhibited tumor growth in A2780 ovarian cancer xenografts but not in A2780 ${ }^{\text {CDDP }}$ ovarian cancer xenografts compared with the untreated control (Fig. 5A, B). However, the combination of HDAC1 shRNA transfection with cisplatin treatment significantly inhibited tumor growth in both ovarian cancer xenografts compared with cisplatin treatment alone (Fig. 5A, B). HDAC1 shRNA did not cause any toxicity or body weight loss during the course of the treatment and up to 6 weeks (data not shown), suggesting that HDAC1 shRNA did not induce any deleterious effects under the present experimental conditions. We subsequently asked the most important question of whether treatment of animals with HDAC1 shRNA, cisplatin, or their combination could effectively target a specific signaling molecule such as HDAC1/c-Myc/miR-34a in tumor tissues. Our results clearly showed that HDAC1 and c-Myc was downregulated and miR-34a was upregulated by HDAC1 shRNA in A2780 ${ }^{\mathrm{CDDP}}$ ovarian cancer xenografts (Fig. 5C-E). Cisplatin treatment alone did not significantly affect HDAC1, c-Myc, or miR-34a expression in A2780 ${ }^{\text {CDDP }}$ ovarian cancer xenografts, but the combined treatment downregulated HDAC1 and c-Myc and upregulated miR-34a (Fig. 5C-E). In A2780 ovarian cancer xenografts, cisplatin treatment alone significantly upregulated HDAC1 and c-Myc expression, and downregulated miR-34a expression (Fig. 5C-E). However, HDAC1 and c-Myc expression was abrogated and miR-34a was restored in tumors upon treatment 


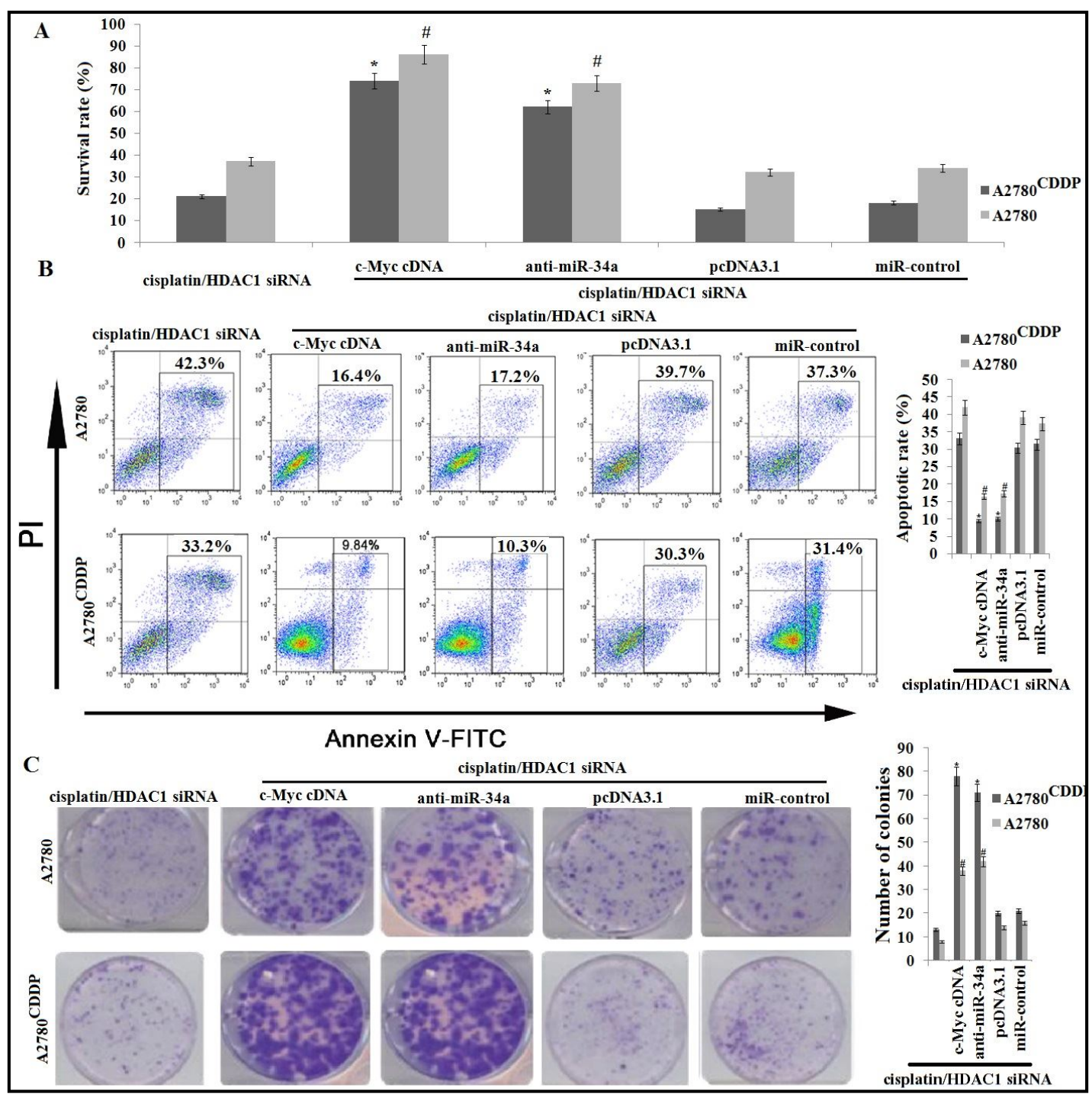

Fig. 4. HDAC1 silencing enhances the sensitivity of $A 2780^{\mathrm{CDDP}}$ or $\mathrm{A} 2780$ cells to cisplatin via the c-Myc/ miR-34a pathway. A2780 ${ }^{\mathrm{CDDP}}$ or A2780 cells were transfected with HDAC1 siRNA or co-transfected with c-Myc cDNA or anti-miR-34a or their control-transfected A2780 ${ }^{\mathrm{CDDP}}$ cells. Then, the cells were treated with cisplatin $(3 \mu \mathrm{g} / \mathrm{mL})$ for $48 \mathrm{~h}$. Cell viability was detected by the methylene blue assay; A, Cell apoptosis was detected using the FACSCalibur flow cytometer; B, The number of colonies formed was detected; C, vs HDAC1 siRNA/cisplatin $\left({ }^{*} \mathrm{P}<0.01,{ }^{\#} \mathrm{P}<0.01\right)$.

with HDAC1 shRNA (Fig. 5C-E). These in vivo results were similar to our in vitro findings, and suggested that inactivation of HDAC1 was at least one of the molecular mechanisms by which cisplatin-induced antitumor activity was potentiated in our experimental animal model. Analyzing tumor apoptosis in situ by TUNEL staining revealed a significantly larger proportion of apoptotic cells in tumors treated with HDAC1 shRNA alone in A2780 CDDP ovarian cancer xenografts, tumors treated with cisplatin alone in A2780 ovarian cancer xenografts, and tumors treated with the combination of HDAC1 shRNA and cisplatin in both cell lines (Fig. 5F). Fewer apoptotic cells were found in A2780 ${ }^{\mathrm{CDDP}}$ ovarian cancer xenografts treated with cisplatin alone, and in A2780 ovarian cancer xenografts treated with HDAC1 shRNA alone (Fig. 5F). 
Fig. 5. Inhibition of HDAC1 sensitizes ovarian cancer cells to cisplatin in a nude mouse xenograft model. A, Growth of tumors in untreated $\mathrm{A} 2780^{\mathrm{CDDP}}$ ovarian cancer xenografts and in $\mathrm{A} 2780^{\mathrm{CDDP}}$ ovarian cancer xenografts treated with cisplatin alone or/and in combination with HDAC1 shRNA untreated in terms of average tumor volume as a function of week of the test; B, Growth of tumors of untreated A2780 ovarian cancer xenografts and in $\mathrm{A} 2780^{\mathrm{CDDP}}$ ovarian cancer xenografts treated with cisplatin alone or/and in combination with HDAC1 shRNA in terms of average tumor volume as a function of week of the test. Arrows represent the time to intraperitoneal injection of cisplatin vs. untreated tumors $\left({ }^{\mathrm{a}} \mathrm{P}<0.05\right.$, ${ }^{\mathrm{b}} \mathrm{P}<0.05$, $\left.{ }^{\mathrm{c}} \mathrm{P}<0.05\right)$; $\mathrm{C}$, Immunohistochemical detection of HDAC1 protein in both cell lines (magnification, $200 \times$ ); D, Immunohistochemical detection of c-Myc protein in both cell lines (magnification, 200×); E, miR-34a expression in both cell lines by the qRT-PCR assay; F, TUNEL staining in a xenograft mouse model (magnification, 200x). vs. untreated tumors $\left({ }^{*} \mathrm{P}<0.05,{ }^{*} \mathrm{P}<0.05\right)$.

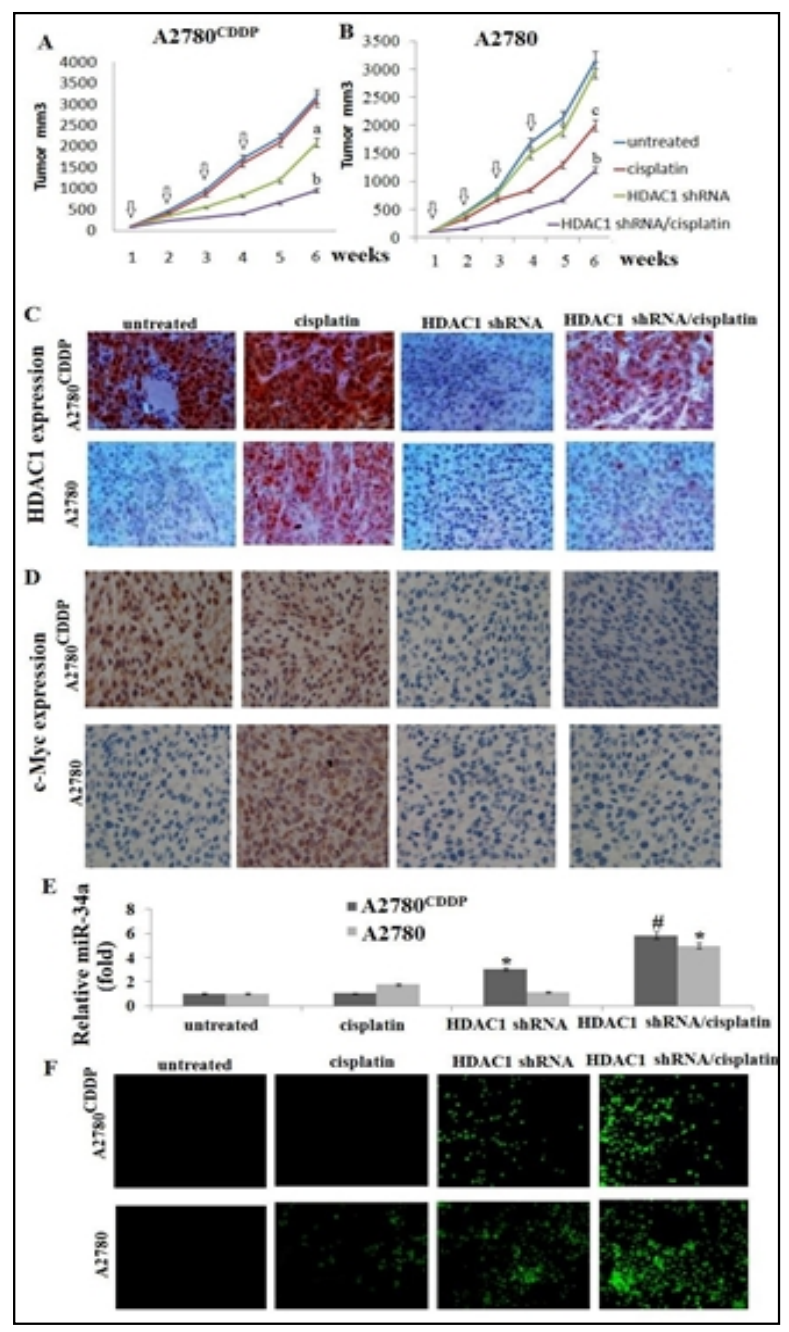

\section{Discussion}

Cisplatin-based chemotherapies are used as first-line treatments for ovarian cancers. Although there is often high responsiveness at first, the majority of patients eventually progress to platinum-resistant disease. HDAC1 expression is associated with drug resistance in several cancers $[10,11,13,33,34]$. Cacan et al [11]. showed that HDAC1 contributes to the suppression of RGS10 during acquired chemoresistance, suggesting that inhibition of HDAC1 may be an adjuvant therapeutic approach to overcome ovarian cancer chemoresistance. In this study, we found high HDAC1 expression in cisplatin-resistant A2780 ${ }^{\text {CDDP }}$ cells, and low HDAC1 expression in cisplatin-sensitive A2780 cells. In addition, inhibiting HDAC1 by siRNA induced apoptosis and inhibited growth of A2780 ${ }^{\mathrm{CDDP}}$ cells but not A2780 cells in vitro and in vivo, indicating that HDAC1 contributes to cisplatin resistance in ovarian cancer cells, and that targeting HDAC1 could restore chemosensitivity in cisplatin-resistant ovarian cancer cells.

We also found that although inhibition of HDAC1 by siRNA alone did not affect cell growth, colony formation, and apoptosis in A2780 cells in vitro and in vivo, HDAC1 siRNA increased the susceptibility of A2780 cells to the cytotoxic action of cisplatin, which is currently used for the treatment of ovarian cancer. Cisplatin treatment significantly induced cisplatin-induced dose-dependent HDAC1 expression in A2780 cells, which caused A2780 cells to acquire chemoresistance to cisplatin. Although A2780 ${ }^{\mathrm{CDDP}}$ cells were resistant to cisplatin, HDAC1 siRNA increased the susceptibility of A2780 ${ }^{\mathrm{CDDP}}$ cells to cisplatin in vitro and 
in vivo. The precise underlying mechanisms by which HDAC1 enhances the chemoresistance of ovarian cancer cells to cisplatin remain unknown.

Evidence suggests the involvement of certain signaling transduction pathways in HDAC1 and cisplatin-induced cancer cell apoptosis. The HDAC1 pathway is activated in many types of cancer and is associated with cancer transformation. It has been shown that miR-34a is positively regulated by c-Myc [30] and that c-Myc is positively regulated by HDAC1 [30]. In this study, we found that targeting HDAC1 induced cell apoptosis and inhibited growth of A2780 ${ }^{\mathrm{CDDP}}$ cells by inhibiting blocking c-Myc expression and inducing miR-34a expression; re-expression of c-Myc decreased miR-34a expression and inhibited HDAC1 siRNA-induced apoptosis of A2780 ${ }^{\mathrm{CDDP}}$ cells. Targeting miR-34a blocked HDAC1 siRNA-induced A2780 cell apoptosis, suggesting that HDAC1 regulates the c-Myc-miR-34a pathway and miR-34adependent apoptosis.

Platinum-based chemotherapy is the standard first-line regimen for advanced ovarian cancer $[35,36]$. The clinical response rate is initially high, but the subsequent relapse and repetitive challenges of chemotherapeutic agents leads to the development of acquired chemoresistance [37]. Such acquired chemoresistance is the major obstacle to the clinical management of ovarian cancer [38, 39]; however, the molecular mechanisms underlying acquired chemoresistance remain largely unknown. In this study, we found that A2780 cells with low HDAC1 expression treated with cisplatin induced dose-dependent HDAC1 upregulation. Co-treatment with HDAC1 silencing and cisplatin increased the cytotoxic effects in both A2780 and A2780 ${ }^{\text {CDDP }}$ cell lines, suggesting that HDAC1 overexpression is the major obstacle for acquired chemoresistance in ovarian cancer cells. These effects were reversed by the re-expression of c-Myc or silencing of miR-34a in both HDAC1 siRNAtransfected A2780 and A2780 ${ }^{\mathrm{CDDP}}$ cells, indicating that HDAC1 silencing sensitizes A2780 and $\mathrm{A} 2780^{\mathrm{CDDP}}$ cells to cisplatin by regulating the c-Myc/miR-34a pathway.

We also examined the effects of transfecting siRNA HDAC1 alone and in combination with cisplatin in preclinical A2780 and A2780 ${ }^{\mathrm{CDDP}}$ models. Targeting HDAC1 by siRNA caused tumor regression in established $A 2780^{\mathrm{CDDP}}$ xenografts but not in A2780, by downregulating c-Myc and upregulating miR-34a. Targeting HDAC1 synergistically enhanced the antitumor activity of cisplatin in both $A 2780^{\mathrm{CDDP}}$ xenografts and A2780 xenografts by downregulating c-Myc and upregulating miR-34a. Combined treatment resulted in greater tumor reduction than each single treatment in the established xenograft models. Taken together, these findings suggest that targeting HDAC1 may be a promising candidate for ovarian cancer therapy as a novel apoptosis inducer with HDAC1-suppressive activity.

In conclusion, the present findings provide evidence of a novel biological function for HDAC1 and a mechanism by which targeting HDAC1 has antitumor effects in cisplatinresistant ovarian cancer cells. In addition, targeting HDAC1 contributes to cisplatin sensitivity in cisplatin-resistant or cisplatin-sensitive ovarian cancer cells in vitro and in vivo. Thus, targeting HDAC1 exhibits antitumor effects and chemosensitivity to cisplatin via the downregulation c-Myc expression and upregulation of miR-34a expression.

\section{Acknowledgements}

The authors wish to thank Professor Da Li for his invaluable scientific contribution and Meifeng Wang for editing the manuscript. This work was supported by grants from the National Natural Science Foundation of China (Grant No: 81402416) and the Strategic Priority Research Programs of the Third Military Medical University (Grant No. TMM130476).

\section{Disclosure Statement}

The authors declare no competing financial interests. 


\section{Cellular Physiology Cell Physiol Biochem 2018;48:1505-1518 and Biochemistry Published online: July 31, $2018 \quad \begin{aligned} & \text { DOI: 10.1159/000492260 } 2018 \text { The Author(s). Published by S. Karger AG, Basel } \\ & \text { www.karger.com/cpb }\end{aligned}$}

Liu et al.: Targeting HDAC1 Sensitizes Ovarian Cancer Cells to Cisplatin

\section{References}

1 Yap TA, Carden CP, Kaye SB: Beyond chemotherapy: targeted therapies in ovarian cancer. Nature Rev Cancer 2009;9:167-181.

2 Jenison EL, Montag AG, Griffiths CT, Welch WR, Lavin PT, Greer J: Clear cell adenocarcinoma of the ovary: a clinical analysis and comparison with serous carcinoma. Gynecol Oncol 1989;32:65-71.

-3 Goff BA, Sainz de la Cuesta R, Muntz HG, Fleischhacker D, Ek M, Rice LW: Clear cell carcinoma of the ovary: a distinct histologic type with poor prognosis and resistance to platinum-based chemotherapy in stage III disease. Gynecol Oncol 1996;60:412-417.

4 Miller M, Ojima I: Chemistry and Chemical biology of taxan anticancer agents. The Chem Record 2001;1:195-211.

5 Haberland M, Montgomery RL, Olson EN: The many roles of histone deacetylases in development and physiology: implications for disease and therapy. Nat Rev Genet 2009;10:32-42.

6 Martin M, Kettmann R, Dequiedt F: Class Ila histone deacetylases: conducting development and differentiation. Int J Dev Biol 2009;53:291-301.

7 Parra M, Verdin E: Regulatory signal transduction pathways for class IIa histone deacetylases. Curr Opin Pharmacol 2010;10:454-460.

8 Khochbin, S, Verdel, A, Lemercier, C, Seigneurin-Berny D: Functional significance of histone deacetylase diversity. Curr Opin Genet Dev 2001;11:162-166.

9 Long J, Fang WY, Chang L, Gao WH, Shen Y, Jia MY, Zhang YX, Wang Y, Dou HB, Zhang WJ, Zhu J, Liang AB, Li JM, Hu J: Targeting HDAC3, a new partner protein of AKT in the reversa of chemoresistance in acute myeloid leukemia via DNA damage response. Leukemia 2017;6:186-193.

10 Cacan E: Histone Deacetylase-1-mediated Suppression of FAS in Chemoresistant Ovarian Cancer Cells. Anticancer Res 2016;36:2819-2826.

-11 Cacan E, Ali MW, Boyd NH, Hooks SB, Greer SF: Inhibition of HDAC1 and DNMT1 modulate RGS10 expression and decrease ovarian cancer chemoresistance. PLoS One 2014;9:e87455.

$\checkmark 12$ Pchejetski D, Alfraidi A, Sacco K, Alshaker H, Muhammad A, Monzon L: Histone deacetylases as new therapy targets for platinum-resistant epithelial ovarian cancer. J Cancer Res Clin Oncol 2016;142:1659-1671.

13 Chen DQ, Pan BZ, Huang JY, Zhang K, Cui SY, De W, Wang R, Chen LB: HDAC 1/4-mediated silencing of microRNA-200b promotes chemoresistance in human lung adenocarcinoma cells. Oncotarget 2014;5:33333349.

14 Kiyoshima T, Yoshida H, Wada H, Nagata K, Fujiwara H, Kihara M, Hasegawa K, Someya H, Sakai H: Chemoresistance to concanamycin A1 in human oral squamous cell carcinoma is attenuated by an HDAC inhibitor partly via suppression of Bcl-2 expression. PLoS One 2013;8:e80998.

15 Sikandar S, Dizon D, Shen X, Li Z, Besterman J, Lipkin SM: The class I HDAC inhibitor MGCD0103 induces cell cycle arrest and apoptosis in colon cancer initiating cells by upregulating Dickkopf-1 and non-canonical Wnt signaling. Oncotarget 2010;1:596-605.

16 Khabele D, Son DS, Parl AK, Goldberg GL, Augenlicht LH, Mariadason JM, Rice VM: Drug-induced inactivation or gene silencing of class I histone deacetylases suppresses ovarian cancer cell growth: implications for therapy. Cancer Biol Ther 2007;6:795-801.

$>17$ Hayashi A, Horiuchi A, Kikuchi N, Hayashi T, Fuseya C, Suzuki A, Konishi I, Shiozawa T: Type-specific roles of histone deacetylase (HDAC) overexpression in ovarian carcinoma:HDAC1 enhances cell proliferation and HDAC3 stimulates cell migration with downregulation of E-cadherin. Int J Cancer 2010;127:1332-1346

18 Cole MD, Nikiforov MA: Transcriptional activation by the Myc oncoprotein. Curr Top Microbiol Immunol 2006;302:33-50.

19 Sabò A, Kress TR, Pelizzola M, de Pretis S, Gorski MM, Tesi A, Morelli MJ, Bora P, Doni M, Verrecchia A, Tonelli C, Fagà G, Bianchi V, Ronchi A, Low D, Müller H, Guccione E, Campaner S, Amati B: Selective transcriptional regulation by Myc in cellular growth control and lymphomagenesis. Nature 2014;511:488492.

20 Baker VV, Borst MP, Dixon D, Hatch KD, Shingleton HM, Miller D: c-myc amplification in ovarian cancer. Gynecol Oncol 1990;38:340-342. 


\section{Cellular Physiology Cell Physiol Biochem 2018;48:1505-1518 \begin{tabular}{l|l} 
DOI: 10.1159/000492260 & $\begin{array}{l}\text { O } 2018 \text { The Author(s). Published by S. Karger AG, Basel } \\
\text { www.karger.com/cpb }\end{array}$
\end{tabular}}

Liu et al.: Targeting HDAC1 Sensitizes Ovarian Cancer Cells to Cisplatin

-21 Reyes-González JM, Armaiz-Peña GN, Mangala LS, Valiyeva F, Ivan C, Pradeep S, Echevarría-Vargas IM, Rivera-Reyes A, Sood AK, Vivas-Mejía PE: Targeting c-MYC in Platinum-Resistant Ovarian Cancer. Mol Cancer Ther 2015;14:2260-2269.

22 Puxeddu E, Durante C, Avenia N: Clinical implications of BRAF mutation in thyroid carcinoma. Trends Endocrinol Metab 2008;19:138-145.

-23 Lodygin D, Tarasov V, Epanchintsev A, Berking C, Knyazeva T, Korner H, Knyazev P, Diebold J, Hermeking H: Inactivation of miR-34a by aberrant CpG methylation in multiple types of cancer. Cell cycle 2008;7:25912600.

24 Wiggins JF, Ruffino L, Kelnar K, Omotola M, Patrawala L, Brown D, Bader AG: Development of a lung cancer therapeutic based on the tumor suppressor microRNA-34. Cancer Res 2010;70:5923-5930.

25 Vinall RL, Ripoll AZ, Wang S, Pan CX, deVere White RW: MiR-34a chemosensitizes bladder cancer cells to cisplatin treatment regardless of p53-Rb pathway status. International J Cancer 2012;130:2526-2538.

26 Li Y, Zou L, Li Q, Haibe-Kains B, Tian R, Li Y, Desmedt C, Sotiriou C, Szallasi Z, Iglehart JD: Amplification of LAPTM4B and YWHAZ contributes to chemotherapy resistance and recurrence of breast cancer. Nat Med 2010;16:214-218.

27 O’Donnell KA, Wentzel EA, Zeller KI, Dang CV, Mendell JT: c-Myc-regulated microRNAs modulate E2F1 expression. Nature 2005;435:839-843.

28 Dews M, Homayouni A, Yu D, Murphy D, , Ripoll AZ, Wang S, Sevignani C, Wentzel E: Augmentation of tumor angiogenesis by a Myc-activated microRNA cluster. Nat Genet. 2006;38:1060-1065.

29 Chang TC, Yu D, Lee YS, Wentzel EA, Arking DE, West KM: Widespread microRNA repression by Myc contributes to tumorigenesis. Nat Genet 2008;40:43-50.

-30 Sotillo E, Laver T, Mellert H, Schelter JM, Cleary MA, McMahon S, Thomas-Tikhonenko A: Myc overexpression brings out unexpected antiapoptotic effects of miR-34a. Oncogene 2011;30:25872594.

-31 Zappasodi R, Cavanè A, Iorio MV, Tortoreto M, Guarnotta C, Ruggiero G, Piovan C, Magni M, Zaffaroni N, Tagliabue E, Croce CM, Zunino F, Gianni AM, Di Nicola M: Pleiotropic antitumor effects of the panHDAC inhibitor ITF2357 against c-Myc-overexpressing human B-cell non-Hodgkin lymphomas. Int J Cancer 2014;135:2034-2045.

-32 Adams CM, Hiebert SW, Eischen CM: Myc Induces miRNA-Mediated Apoptosis in Response to HDAC Inhibition in Hematologic Malignancies. Cancer Res 2016;76:736-748.

-33 Johnson DP, Spitz GS, Tharkar S, Quayle SN, Shearstone JR, Jones S, McDowell ME, Wellman H, Tyler JK, Cairns BR, Chandrasekharan MB, Bhaskara S: HDAC1, 2 inhibition impairs EZH2- and BBAP-mediated DNA repair to overcome chemoresistance in EZH2 gain-of-function mutant diffuse large B-cell lymphoma. Oncotarget 2015;6:4863-4887.

-34 Lin YC, Lin YC, Shih JY, Huang WJ, Chao SW, Chang YL, Chen CC: DUSP1 expression induced by HDAC1 inhibition mediates gefitinib sensitivity in non-small cell lung cancers. Clin Cancer Res 2015;21:428-438.

-35 Agarwal R, Kaye SB: Ovarian cancer, strategies for overcoming resistance to chemotherapy. Nat Rev Cancer 2003;3:502-516.

-36 Geisler JP, Manahan KJ, Wiemann MC: Chemotherapy for ovarian cancer: an evidence-based approach. Minerva Ginecol 2004;56:539-545. Gottesman MM: Mechanisms of cancer drug resistance. Annu Rev Med 2002;53:615-627. Cannistra SA. Cancer of the ovary. N Engl J Med 2004;351:2519-2529.

-39 Zeller C, Dai W, Steele NL, Siddiq A, Walley AJ, Wilhelm-Benartzi CSM, Rizzo S, van der Zee A, Plumb JA, Brown R: Candidate DNA methylation drivers of acquired cisplatin resistance in ovarian cancer identified by methylome and expression profiling. Oncogene 2012;31:4567-4576. 